\title{
Pain polymorphisms and opioids: An evidence based review
}

\author{
CLÁUDIA MARGARIDA PEREIRA VIEIRA ${ }^{1-3}$, ROSA MARIA FRAGOSO ${ }^{1,4}$, \\ DEOLINDA PEREIRA $^{1}$ and RUI MEDEIROS ${ }^{2,3,5,6}$ \\ ${ }^{1}$ Medical Oncology Department; ${ }^{2}$ Research Centre-Molecular Oncology Group, \\ Instituto Português de Oncologia do Porto Francisco Gentil (IPO-PORTO), 4200-072 Porto; \\ ${ }^{3}$ Faculty of Medicine, University of Porto, 4200-319 Porto; ${ }^{4}$ Unit of Study and Treatment of Pain, \\ Instituto Português de Oncologia do Porto Francisco Gentil (IPO-PORTO), 4200-072 Porto; \\ ${ }^{5}$ Biomedical Research Center, Faculty of Health Sciences, Fernando Pessoa University, 4249-004 Porto; \\ ${ }^{6}$ Research Department, Portuguese League Against Cancer, 4200-172 Porto, Portugal
}

Received April 22, 2018; Accepted September 20, 2018

DOI: $10.3892 / \mathrm{mmr} .2018 .9792$

\begin{abstract}
Despite the various different candidate genetic polymorphisms of potential clinical relevance, there is not enough understanding of the inter-individual variability in analgesic administration. The cytochrome P450 2D6 (CYP2D6) genotype is thought to be one of the most studied. The aim of the present evidence-based review was to determine if there is now sufficient evidence to make clinical recommendations based on a specific genomic profile. The data sources utilized were as follows: PubMed (NLM) database, Evidence Based Medicine Guidelines and Google. Research on clinical guidance standards, systematic reviews, meta-analyses and clinical trials, published prior to January 2018, were evaluated in English, using the MeSH terms 'cancer pain', 'polymorphism', 'genetic' and 'gene polymorphism'. To assess the level of evidence, the Strength of Recommendation Taxonomy of the American Family Physician was applied. From the initial search, 12 systematic reviews and/or meta-analyses, 5 clinical trials and 10 guidelines were selected. The results indicated that genetic variation of $\mu$-opioid receptor 1 (OPRM1) may contribute to inter-individual differences in morphine consumption with recommendation grade A for OPRM A118G single nucleotide polymorphism (rs1799971). Polymorphisms associated with the metabolization process of morphine and other opioid drugs are very relevant in opioid titration and ethnic subgroup differences which have to be taken into account (particularly, for the recommendation grade A for the CYP2D6 polymorphism). In human studies, the catechol-O-methyl transferase (COMT)
\end{abstract}

Correspondence to: Dr Cláudia Margarida Pereira Vieira, Medical Oncology Department, Instituto Português de Oncologia do Porto Francisco Gentil (IPO-PORTO), Rua Dr António Bernardino de Almeida, 4200-072 Porto, Portugal

E-mail: claudia.vieira@ipoporto.min-saude.pt

Key words: polymorphism, opioid, cancer pain, genetic, gene, analgesics, pharmacogenetics, single nucleotide polymorphism genotype affects the efficacy of opioids in acute and chronic pain under different settings, with recommendation grade $\mathrm{B}$ to the COMT single nucleotide polymorphism rs4680 (Val/Met). Finally, polymorphisms of the ATP-binding cassette family of efflux transporters were highlighted. Consistent data on pain polymorphisms is now widely available; however, these results have had very little impact on clinical guidelines and daily oncologist practice. Persisting pain, side effects of grade 3 (NCI-CTCAE v4.0) and breakthrough pain with more than 4 episodes/day should be considered the criteria for pain multidisciplinary team discussions and for polymorphism screening.

\section{Introduction}

Chronic pain inadequately treated is a major public health issue (1). This is particularly relevant, when we are talking of supportive care in cancer patients. Opioids are the most used analgesics for cancer pain, but the clinical benefits of opioid analgesics are dependent of substantial individual variations in the responses to opioids, insufficient drug dosing and/or a high rate of adverse events. The wide interindividual variability in sensitivity to opioids leads to unpredictable clinical responses to opioid treatment and adverse events, along with narrow therapeutic window and are still nowadays an important problem $(1,2)$.

To date, only a limited number of studies have addressed the relationship between human genetic variations and sensitivity to opioids; however there is growing evidence that pharmacogenetic differences may impact in interindividual variability in opioid response. Human genetic variation may directly modulate opioids pharmacokinetic and pharmacodynamic effects; candidate genes are sought in polymorphisms of drug transporters, metabolizing enzymes or opioid receptors $(1,2)$.

Cytochrome P450 (CYP) are enzymes located on the smooth endoplasmic reticulum membranes of liver hepatocytes and along the mucosal surface of the intestinal tract. The CYP system can inactivate or activate a given drug (type I reactions) and is responsible for glucuronidation and sulfation, connected with drug excretion (type II). Along with CYP iso-enzyme 3A4 
(CYP3A4), the most important enzyme is CYP 2D6 (CYP2D6). She is involved in the metabolism many drugs used in pain and palliative medicine (e.g., opioids, neuroleptics, antidepressants). More than 80 distinct allelic variants for CYP2D6 are known, which leads to a wide spectrum of metabolic capacity and phenotype diversity within populations for several drugs like tramadol, dihydrocodeine, codeine (3). Fentanyl is thought to be predominantly metabolized in the liver by CYP3A4-mediated $\mathrm{N}$-dealkylation (less than $1 \%$ is metabolized by alkyl hydroxylation, $\mathrm{N}$-dealkylation or amide hydrolysis) (4). Ketamine is mainly metabolized by CYP2B6 and CYP3A4 (5). Morphine is metabolized to morphine-3-glucuronide (M3G) and morphine-6-glucuronide (M6G) via glucuronidation by phase II metabolism of UDP-glucuronosyl transferase 2B7 (UGT2B7). About $60 \%$ of morphine is converted to $\mathrm{M} 3 \mathrm{G}$ and $6-10 \%$ is converted to M6G. Both metabolites are excreted in the urine. M6G is a very potent opioid analgesic, which activates $\mu$-opioid receptors, while M3G has no opioid properties and has been proposed to be responsible for neuroexcitatory effects, including allodynia, myoclonus and seizures. Two variants of the UGT2B7 gene have been described, with inconsistent results on their influence on morphine glucuronidation and pain relief (6).

The opioid pharmacogenetic studies in cancer patients have primarily focused on three genes: $\mu$-opioid receptor (OPRM), catechol-O-methyl transferase (COMT) and multidrug resistance 1 gene (MDR-1) (7).

$\mathrm{Mu}$-opioid receptors (MOR) receptors are the main site of action of opioids. Recent genetic research shows that genetic variations in $\mu$-opioid receptor 1 (OPRM1) gene locus play an essential role in inter-individual responses. This may explain why some patients do need higher doses of opioid for pain relief, translating into decreased morphine potency in pupil constriction and experimental analgesia, or poor receptor signaling in vitro (8). Numerous single nucleotide polymorphisms (SNPs) in the mu opioid receptor have been identified, but the majority of genetic association studies have focused on the $\mathrm{A} 118 \mathrm{G}$ polymorphism ( $\mathrm{A}>\mathrm{G}$ functional substitution at locus 118,) which codes for a non-synonymous change in OPRM1 exon $1(9,10)$.

Another line of evidence indicates that the influence of COMT gene polymorphisms on pain has also been investigated. It has been shown that the Val158Met polymorphism, a common genetic variant in Caucasian populations, influences the activity of the COMT enzyme. This enzyme, which metabolizes dopamine, adrenaline and noradrenaline, is an important modulator of dopaminergic and noradrenergic neurotransmission, known to play a role in pain (11-13).

Furthermore, functional impairment of peripheral blood morphine transporters (multi-drug resistance protein, proteins 2 and 3; -MRP2 and MRP3 genes), and morphine transporters through the blood-brain barrier, like ATP-binding cassette (ABC) family of efflux transporters, may result in modification of brain's morphine concentration (14).

There are a growing number of candidate genes for genetic polymorphisms of potential clinical relevance (not all referred in this paper). The aim of this study is to determine if there is now enough evidence to make treatment recommendations based on specific genomic profile in cancer pain patients. In the future, a faster titration of opioid needs would be possible, with less episodes of irruptive pain or persistent pain, fewer side effects and thus better quality of life. We would probably have a lower incidence of chronic pain. Identifying patients' subgroups more susceptible to refractory pain or adverse symptoms would give us the ability to anticipate cases of difficult pain control, with better pain control, fewer visits and hospitalizations for uncontrolled pain or adverse events and lower costs; pain control could increase cost-effectiveness.

\section{Materials and methods}

A bibliographic survey was carried out in the following databases: PubMed (NLM), Evidence Based Medicine Guidelines and Google. Clinical guidelines, systematic reviews, metaanalyzes and clinical trials, published until January 2018, were searched using MeSH terms 'cancer pain', polymorphism, genetic, gene polymorphism. Similar search strings were adapted for the others databases. In Google, search was also conducted by organization with particularly dedication to cancer pain issues. Only guidelines in English, which were published and downloadable from the web, were taken into consideration. Exclusion criteria in the selection of articles were: papers without any reference to polymorphism (or genetic variation) in the abstract, reporting drug use in non-cancer pain or being related to nursing practice or anti-cancer therapies or translational pain research (animal models).

The American Family Physician's (AFP) Strength of Recommendation Taxonomy (15) was applied to classify levels of evidence and recommending strengths. According to this taxonomy, the quality of the study is subdivided into three Levels of Evidence (Level of Evidence 1: Good quality studies, evidence-oriented decision; Level of Evidence 2: Limited quality studies, patient-oriented evidence; Level of Evidence 3: Other evidence) and the Strength of Recommendation in also divided in three levels-(Strength of Recommendation A: consistent, evidence patient-oriented; Strength of Recommendation B: Inconsistent or limited quality, evidence oriented for the patient; Strength of Recommendation C: consensus, evidence-oriented disease). The final text has been reviewed and approved by all authors.

\section{Results}

The search for polymorphism (42 papers) or genetic (86 papers) or gene polymorphisms (34 papers) in patients with "cancer pain' identified 35 systematic review and/or meta-analysis and 12 clinical trials. More than half of the articles were excluded because they did not concern systematic review and/or meta-analysis and clinical trials or they did not meet the inclusion/exclusion criteria (Fig. 1). For these analyses 12 systematic review and/or meta-analysis and 5 clinical trials were selected. The search for Clinical Guidance Standards found 10 guidelines with reference to pain polymorphism or genetics of pain (from a total of 38 found in our search).

Clinical guidance standards. We found at least 38 guidelines on supportive care and pain but reference to pain polymorphism, or the need to search for any particular polymorphism at the individual at level, was absent in the great majority; some have a generic reference to this subject. 


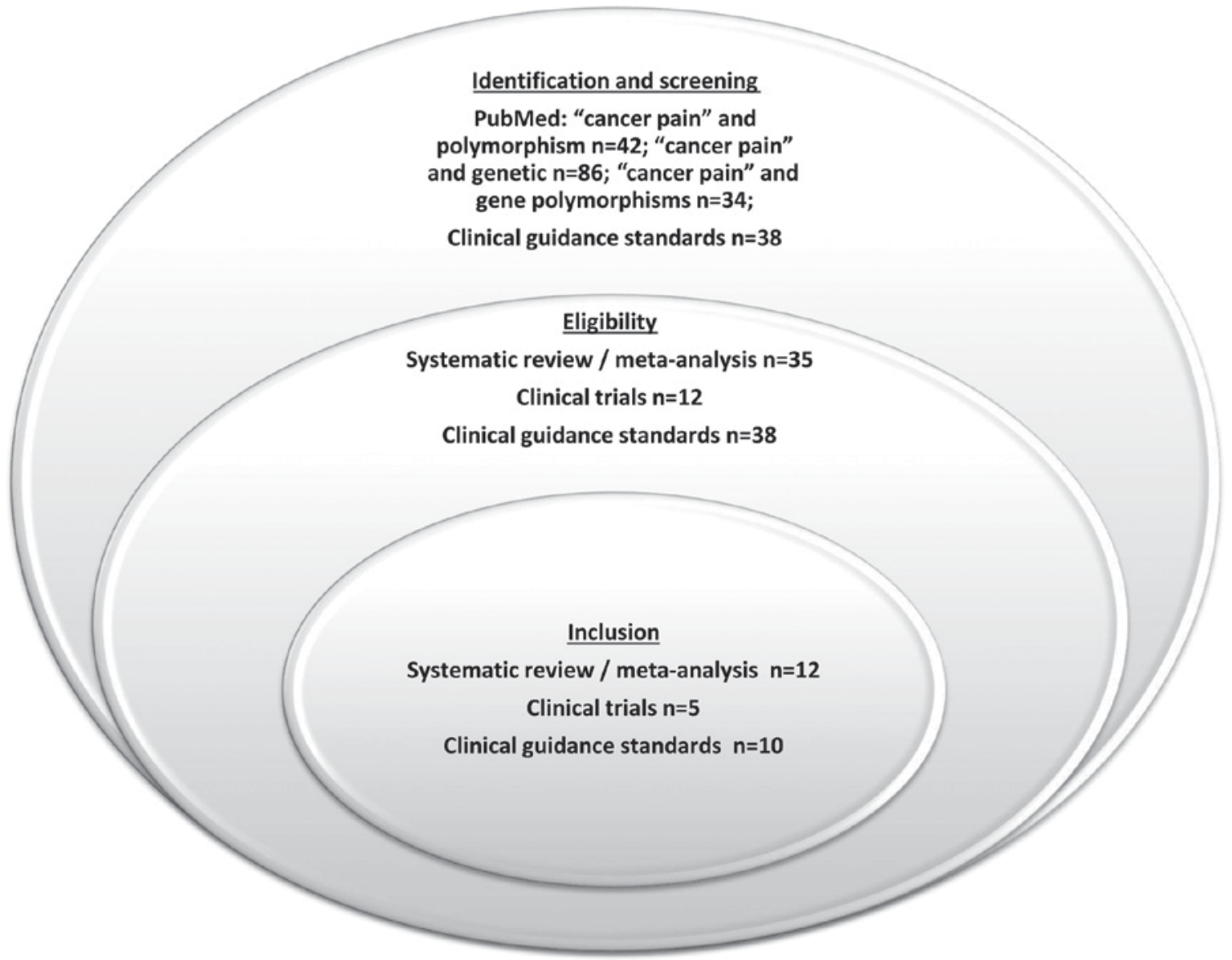

Figure 1. Flow diagram of the methods employed for the identification and selection of relevant articles included in the present evidence-based review.

The genetics associated with pain arises, as in the position paper promoted by the European Pain Federation in January 2017 (2), as an explanation for the marked inter-individual variability in responsiveness to different opioids, both in terms of analgesic benefit and toxicity $(2,16)$. This paper refers to genetic variability in $\mu$-opioid receptors (central and peripheral), with different binding affinities of the opioids, and additional $\mathrm{k}$ - and $\delta$-opioid receptors to explain the need for individualization of pain treatment, both in terms of response to treatment and adverse events. However, authors cite, without further specification, that other molecular, pharmacological, genetic and phenotypic factors may explain the variation in observed clinical responses (2).

The National Comprehensive Cancer Network (NCCN) guidelines (January 2018) recognizes that different polymorphisms in CYP2D6, as in some ethnic group, may justify the existence of either slow or fast metabolizers of codeine (17). Codeine is a weak $\mu$ and $\delta$-opioid receptor agonist with little direct analgesic effect. The action of the cytochrome P450 enzyme-CYP2D6-is necessary to convert the prodrug into active metabolites (codeine-6-glucuronide, norcodeine, morphine, morphine-3-glucuronide, morphine-6-glucuronide and normorphine). The poor/slow metabolizers (five to ten per cent of the population) will obtain reduced or no analgesic effects and the hyper-metabolisers metabolizers (five to ten percent) may experience more rapid morphine production with increased risk of toxicity. There is considerable inter-ethnic variability in gene encoding for CYP2D6 $(17,18)$.
The European Palliative Care Research Collaborative opioid guidelines project endorsed by MASCC (Multinational Association of Supportive Care in Cancer, also refers that approximately $8 \%$ of the European population are poor metabolizers of codeine to morphine, with resulting diminished analgesic efficacy. Also genetic polymorphisms, with impact in O-demethylation (via CYP2D6) can lead to alterations in response to tramadol in a similar way to codeine. The active metabolite, O-desmethyltramadol, has a higher affinity to the $\mu$-opioid receptor, than the parent drug, however in vivo; production seems to be slow with minimal clinically relevant accumulation (19-21). Searching for this profile is not done routinely, despite the reference in the NCCN and WHO guidelines $(17,22-24)$. In addition to the analgesic effect, codeine is probably used more often as antitussive than analgesic, in cancer patients $(17,20-25)$. These authors suggest that the great interindividual variation in the amount and ratios of metabolite production may not be all accounted for by known polymorphisms. For instance the CNS adverse effects have been shown to occur even in the absence of significant CYP2D6 activity, suggesting a potential role for metabolites other than morphine in toxicity $(17,20,21,25)$.

The World Health Organization (WHO) guideline explains that both codeine and tramadol may be less analgesic in poor metabolizers (22-24). Tramadol is also extensively metabolized in the liver by demethylation, oxidation and conjugation (2-4). The main active metabolite, O-demethyl-tramadol (M1), is the result of the catalytic action of CYP 2D6 and is two to 
four times more potent than the parent compound. The poor metabolizers have 14 -fold lower concentrations of the active metabolite and may have less analgesic efficacy (22-25). Schug et al (26) based on WHO guideline proposed in Expert opinion-Pain management of the cancer patient-that polymorphisms in CYP2D6 result in a range of metabolic patterns, from ultrarapid to ultra-slow metabolizers of codeine, with some patients showing no analgesic effect at all. This paper also refers that genetic variability in morphine metabolism may have a role in neurotoxic and hyperalgesic effects of $\mathrm{M} 3 \mathrm{G}$, but also with the influence of genetic variants of the OPRM1 gene, encoding the $\mu$-opioid receptor (26).

The Centers for Disease Control and Prevention (CDC) published the CDC Guideline for Prescribing Opioids for Chronic Pain clarifying that equianalgesic dose conversions are only estimates and do not account for individual variability in genetics and pharmacokinetics. This fact is particularly relevant to patients' members of racial and ethnic minority groups that can be at risk for inadequate pain treatment (27).

Also the Canadian or Scottish guidelines support that conversion ratios for opioids are subject to variations in kinetics governed by genetics and other drugs. Even with the same chronic pain syndrome, the underlying neurobiology will differ between individuals, influencing analgesic response and side effects $(18,28)$. Studies into factors that contribute to the inter-individual variation in response to different treatments (clinical, genetic, pharmacokinetic, neurobiological) and clinical biomarkers for predicting response to treatments are still needed. Another factor that must be considered, when assessing opioid responses, is that several opioids including codeine, tramadol, oxycodone and hydrocodone are affected by variations in metabolism, mediated by cytochrome $\mathrm{P} 450$ enzyme CYP2D6, resulting in unpredictable effects in individuals $(18,28)$.

Pharmacological Management of Cancer Pain in Adults-National Clinical Guideline No. 9 was promoted by Ireland experts and classified with level 5 of evidence (based on the Centre for Evidence Based Medicine method of Oxford University) the role of CYP2D6 enzyme inhibitors or genetic polymorphisms in morphine sulphate production with reduced analgesic response. This poor or absent analgesic effect of codeine can affect approximately $7 \%$ of Caucasian people, $3 \%$ of black people and $1 \%$ of Asian people (29). Wide inter-individual variability in opioid pharmacokinetics is influenced by genetic variation but also by age, ethnicity and the presence of renal or hepatic impairment $(29,30)$. Further prospective research may allow prediction of inter-individual response to different opioids and better opioid prescribing (27).

Summary, we can say that the guidelines are divided in 3 groups: One first group with reference to the route of metabolization of cytochrome P450 enzyme CYP2D6; a second group that's presents a generic reference to the impact of the polymorphisms on pain treatment, without specifying any one; and a last group (not exhaustively referred to in this work) that's does not present any reference to this matter.

Systematic review and/or meta-analysis. The search identified 35 systematic review and/or meta-analysis, but only 12 had direct reference to some specific polymorphism (Table I). Three reviews were excluded because they don't have any reference to polymorphism (or genetic variation) in the abstract, five articles was only available in Japanese, Chinese or German language and three was reporting drug use in chronic non-cancer pain and the others was related with nursing practice or anti-cancer therapies or translational pain research (animal models). Data summarized in Table I.

These papers try to summarize and value the functional impact of several genotype groups/reference SNP identification. Principals polymorphism referred in this literature review and the strength of recommendation for pain polymorphisms are discussed below.

Clinical trials. The search identified 12 clinical trials, but only five had direct reference to some specific polymorphism in cancer patients (Table II). Clinical trials were related with polymorphisms in CYP2B6 (metabolism of ketamine), CYP2D6 (analgesia of tramadol) and UGT2B7 (morphine). One trial was related with single nucleotide polymorphism at nucleotide position 118 in the $\mu$-opioid receptor gene. Another reported pain outcomes and genetic variation was analyzed for 112 single nucleotide polymorphisms (SNPs) in 25 candidate genes relevant for opioid efficacy. The choice of the candidate SNPs was based on the expected clinical relevance of the variant alleles (allele frequency $>0.10$ ), previously described associations or putative functional effects related to pain and opioid pharmacology. The relevance of this papers and main findings are discussed below.

\section{Discussion}

Revealing the relationship between genetic variations and individual differences in sensitivity to opioids will provide valuable information, for appropriate individualization of opioid doses required for adequate pain control. This evidence based review identifies the most promising polymorphism in the cancer pain treatment. However, application of this knowledge to clinical practice, creating easier to use diagnostic tools is more difficult to achieve (31).

Several candidate genes have been used to provide evidence for the genetic modulation of pain perception and response to analgesics. However due to the limited number of patients in prospective trials, the several number of genes and genetic variants investigated and the lack of clinical randomized trials, the level of evidence is in general low. Precision medicine and personalized analgesic treatments will require a more complete understanding of the effects of genetic variants and gene-gene interactions in response to analgesics (32).

According to our evaluation, three groups of polymorphism were highlighted in this research (Fig. 2; Table III). Firstly, the genetic variation of the $\mu$-opioid receptor may contribute to interindividual differences in morphine consumption. In the future, identifying single nucleotide polymorphisms of patients may provide information to modulate the analgesic dosage of opioid for faster achievement of satisfactory pain control $(33,34)$. The mu opioid peptide receptor (MOP) is the principal site of pharmacologic actions for most clinically important opiate drugs, but there are more than 100 polymorphisms identified in the human $\mu$ opioid peptide receptor (OPRM1) gene. These polymorphisms correlated with OPRM1 mRNA stability and opiate sensitivity, including 


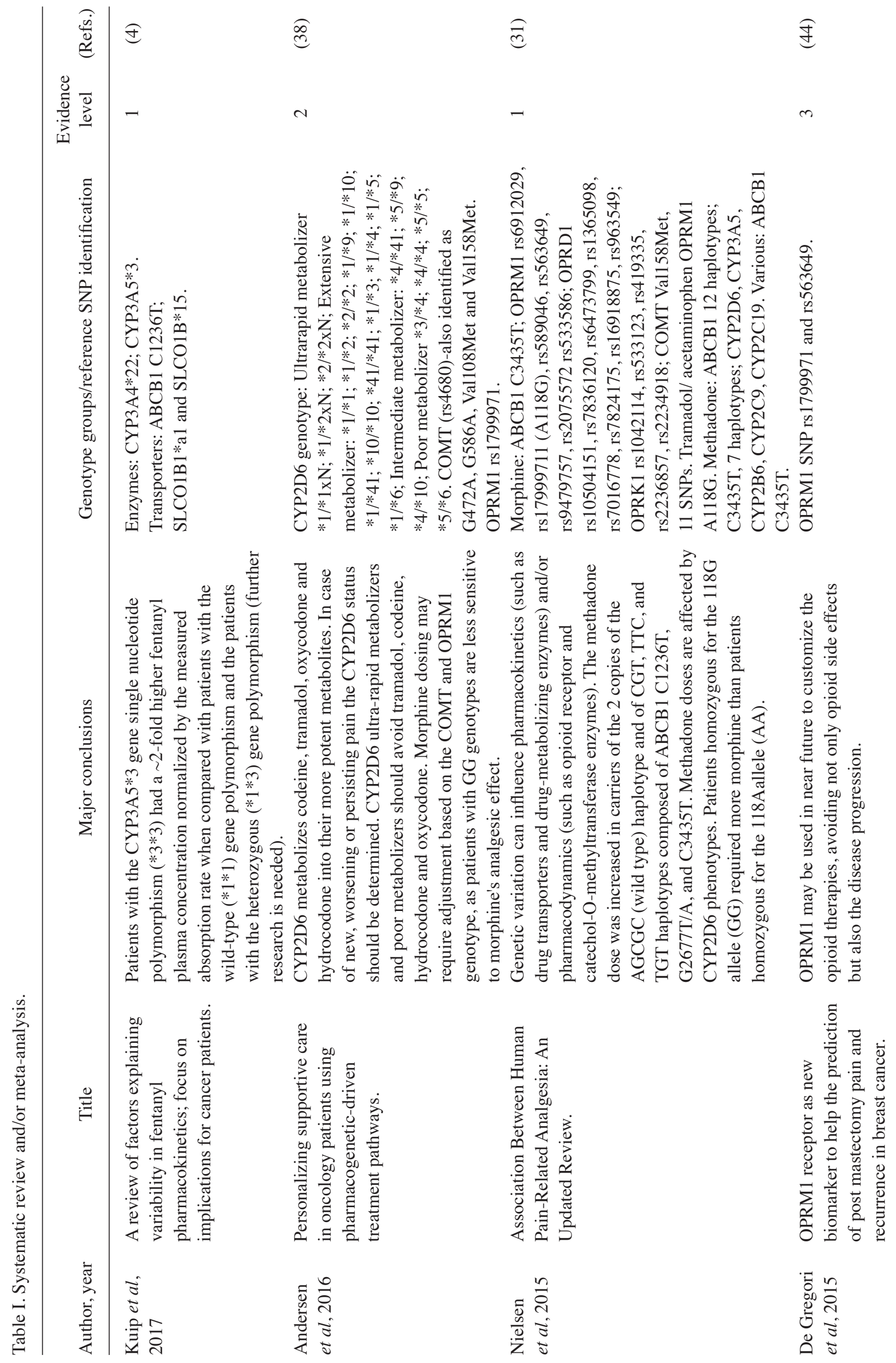




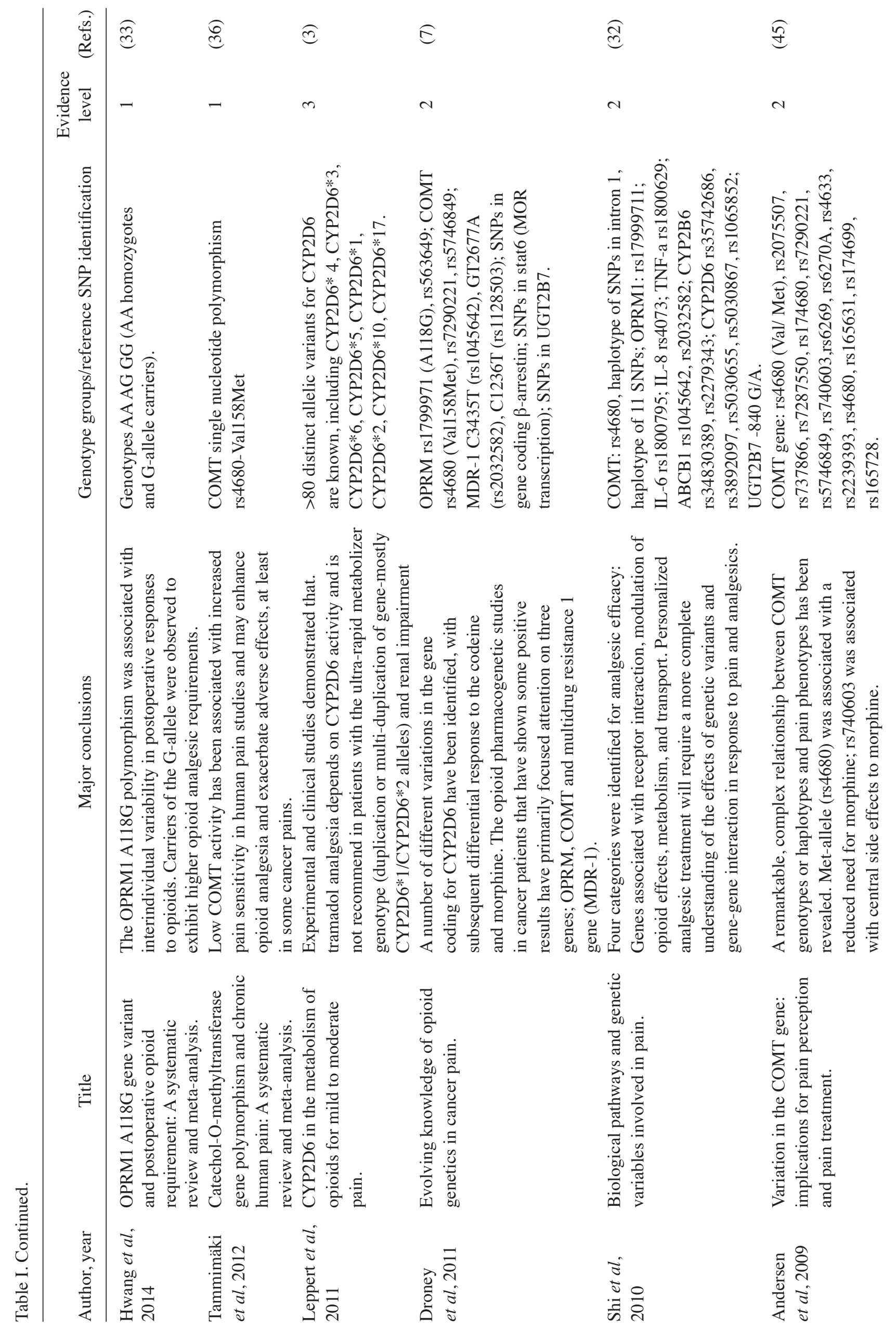


opiate analgesia, tolerance and dependence. Particularly relevant is OPRM1 A118G polymorphism rs17999711 (33,34). In addition to the A118G polymorphism, another functional SNP (rs563649), which is located within an alternatively-spliced OPRM1 isoform (MOR-1K), has also has been the subject of several work $(7,8)$. More precise studies are need to better understanding the relationship between gene polymorphisms and opiate sensitivity, that will allowed personalized pain treatment, by predicting opiate sensitivity and requirements for each patient (35).

Secondly, polymorphism associated to the metabolization process of morphine and other opioid drugs, manly polymorphism of CYP enzymes, are nowadays very relevant in opioid titration and rotation. Also referenced in the literature are genetic polymorphisms in genes of uridine diphosphate glucuronosyltransferase (UGT) enzymes. Ethnical and population subgroups differences have to be taken in account. Prospective trials of cancer patients and healthy controls are need, at a national or regional level, to identify these subgroups (31). Catechol-O-methyltransferase (COMT) is one of several enzymes that metabolize catecholamines. In human studies, COMT genotype affects the efficacy of opioids in acute and chronic pain in different settings (e.g. migraines, fibromyalgia, musculoskeletal pain and cancer pain). Low COMT activity increases opioid receptors and enhances opioid analgesia and adverse effects in cancer pains. Pain animal models had elucidated the mechanism behind these findings: COMT inhibitors are pronociceptive, except for neuropathic pain. The complex network between adrenergic and dopaminergic activity in different parts of the nociceptive system may have a role in the action of low COMT activity (36).

Finally, ATP-binding cassette (ABC) family of efflux transporters consists of around 50 human members. However ABCB1 (MDR1) is the most well characterized, coding for the P-glycoprotein (P-gp) efflux transporter (31).

Among all, only two of them satisfied the proposed criteria as A level of recommendation: OPRM1 polymorphism A118G (rs1799971) and polymorphisms in cytochrome P450 enzyme-CYP2D6 (Table III). These polymorphisms were studied in randomized trials and are extensively referred in the systematic reviews and meta-analyzes. An algorithm of management of pain and polymorphism screening is proposed in the Fig. $3(17,19,37)$. Actual guidelines make some appointments about these genetic variations, as responsively for interindividual differences in the response to opioids, yet almost all without, without proposed a real clinical setting to use them $(17,38,39)$.

It is important to remind, that a combined effect of SNPs in multiple genes is possible and its investigation, at the same time, should remain a concern for prospective studies in this field. And also take in consideration, the role of environmental factor in interpatient variability in responses to opioids. Information on type of SNPs (intronic, exonic, or intergenic) was not present in the majority of these papers. Only two papers refered to intronic SNPS in COMT (rs7290221, rs5746849, rs4646312, rs6269 and rs740603). Usually intronic SNPs are not drivers, but could be functional by changing the conformation of RNA and DNA neighboring the SNPs (7,40-42). 


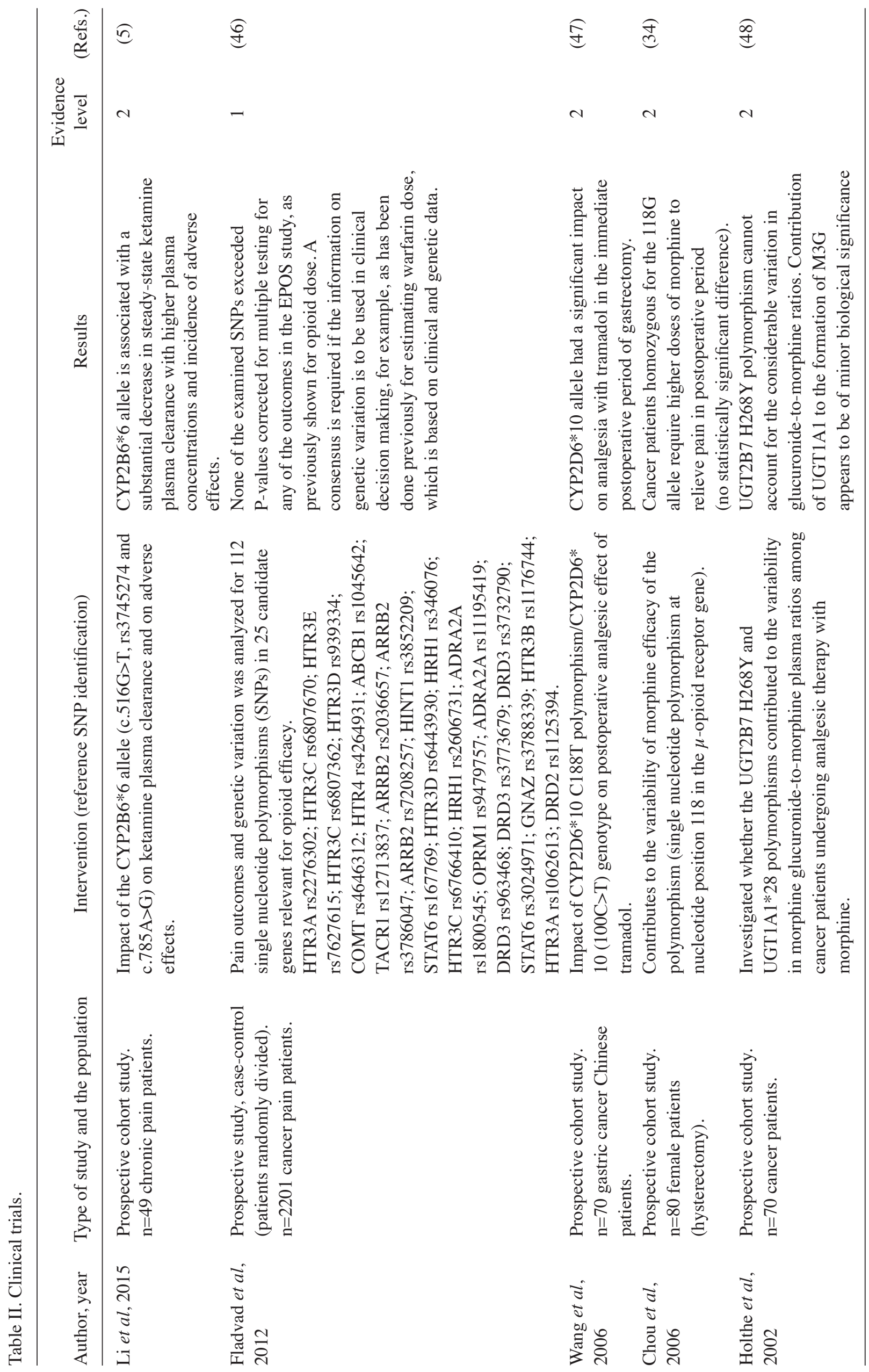


Table III. Strength of recommendation for pain polymorphisms.

Recommendation

Polymorphism

Impact

level

$\mu$-opioid receptors (central and peripheral):

Individualization of pain treatment in terms of response to

A

OPRM1 polymorphism A118G (rs1799971) treatment and adverse events. If OPRM1 G/G genotype consider initiating morphine at a higher dose and/or more aggressive dose titration. May also influence tramadol/acetaminophen analgesic response.

Polymorphism in cytochrome P450 enzyme: Several opioids metabolisms are affected (codeine, tramadol, CYP2D6 genotype (particularly CYP2D6*10 oxycodone, hydrocodone, methadone). Poor metabolizers allele/C188T) may be at risk of treatment failure due to the inability to convert the parent drug into its more active metabolite.

Ultra-rapid metabolizers may be at risk of treatment-related toxicities to supratherapeutic concentrations of the more active metabolites.

Polymorphism in catechol-O-

Low COMT activity (Val158Met) can increase opioid

methyltransferase (COMT) enzyme: Single nucleotide polymorphism rs4680)

(Val158Met) receptors and enhance opioid analgesia and adverse effects. If COMT G/G genotype, consider initiating morphine at a higher dose and/or more aggressive dose titration.

Polymorphism in cytochrome P450 enzyme: Variations in metabolism of ketamine.

CYP2B6*6 allele (c.516G $>$ T, rs3745274

and c. $785 \mathrm{~A}>\mathrm{G})$

Polymorphism in cytochrome P450 enzyme: May influence fentanyl pharmacokinetics.

CYP3A5*3 gene single nucleotide polymorphism

Polymorphism in ABCB1 (MDR1): C1236T, Methadone doses are subject to ABCB1 genetic modulations. G2677T/A and C3435T C3435T polymorphism (variant T allele) may influence dose requirements for others opioids (e.g. morphine).

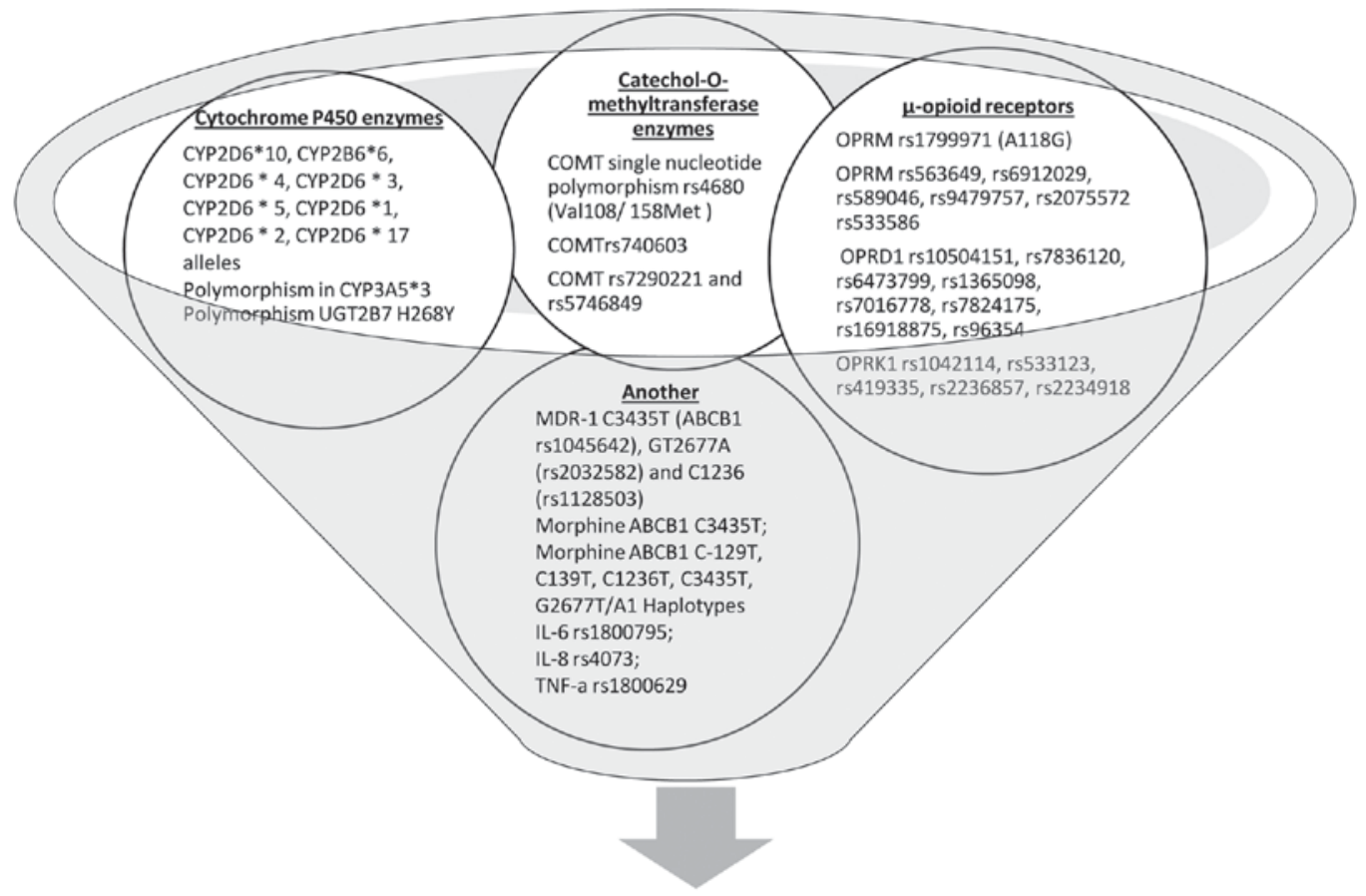

OPRM1 receptor polymorphism A118G (rs 179997)

Polymorphism in cytochrome P450 enzyme - CYP2D6 alleles

COMT single nucleotide polymorphism rs 4680 (Val158Met)

Figure 2. Principals polymorphisms referred to in the present literature review. 
Moderate/ severe pain or persisting mild/ moderate pain despite analgesic treatment
Strong opioids +/NSAIDs/ paracetamol +/adjuvant drugs
Persisting pain, despite increasing doses of opioid, correct titration and opioid/ route of opioid switching (if necessary)

or

side effects grade 3

or

more than 4 episodes of breakthrough pain per day
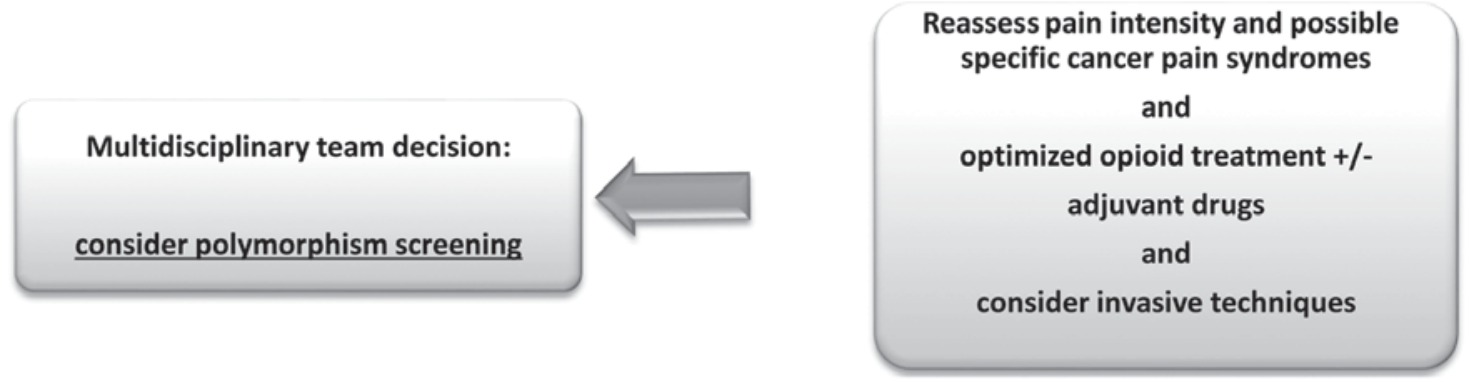

Figure 3. Proposed algorithm for the management of pain and polymorphism screening. NSAIDs, non-steroidal anti-inflammatory drugs.

The clinical utilization in daily practice is also much dependent of the cost of time and laboratory resources of these analyses (38). Pain is an oncology emergence and pain drugs titration may be incompatible with delayed laboratory tests and very expensive polymorphism determinations. Frequently the allocation of technical and economic resources to anti-cancer treatment limits the evolution of these strategies for better individualized supportive and palliative care treatment. Resources are currently limited and a global balance is needed for fairer and more equitable treatment worldwide. The identification of population or ethnic subgroups more prone to poor pain control may lead to a strategy of lowering the costs of the treatments. However, an investment in the development of laboratory tests for the rapid identification of these polymorphisms and an exhaustive training for health professionals is still an unmet need.

One limitation of this work is the criteria for the literature search, particularly the limitation to clinical guidelines, systematic reviews, meta-analyzes and randomized clinical trials in English. This evidence-based review is not a systematic review, in the strict sense of the term, because they do not include reviews and systematic reviews and they need a different frame of search terms and analyses. Furthermore our goal was to apply the levels of evidence and recommending strengths of The American Family Physician's (AFP) Strength of Recommendation Taxonomy (15).

There is considerable inter-ethnic variability in gene encoding for CYP2D6. The differences between countries and the ethnic variations may result in loss of date not published in English or published in some national journals. Data from retrospectives series or no randomized trials can have some importance in this field and should be addressed in a future paper. A statistical meta-analysis, with the strength of the associations of the SNPs with pain score, is also a future project. Other aspect to take in account, is the bias frequent found on this kind of research. The papers summarized in the systems reviews and meta-analyzes try to highlight differences in pain prevalence and treatment conditioned by the polymorphism or other genetic alterations, however it's difficult to eliminated the effect of numerous others factors, with influence on human pain, and specially cancer pain.

An algorithm of management of pain and polymorphism screening is proposed and three groups of polymorphism are considered of relevance for present utilization on clinical practice. Genetic variation of the $\mu$-opioid receptor may contribute to interindividual differences in morphine consumption (with recommendation grade A for OPRM A118G rs1799971) but there are more than 100 polymorphisms identified in the human $\mu$ opioid peptide receptor (OPRM1) gene. Polymorphism associated to the metabolization process of morphine and other opioid drugs are nowadays very relevant in opioid titration and rotation. Ethnical and population subgroups difference have to be taken in account. A recommendation grade A was awarded for polymorphism in cytochrome P450 enzyme-CYP2D6). In human studies, COMT genotype affects the efficacy of opioids in acute and chronic pain in different settings, with recommendation grade B to COMT single nucleotide polymorphism rs 4680 (Val158Met).

Consistent data on pain polymorphism is nowadays available, however with very little impact on clinical guidelines and daily oncologist practice. Persisting pain, side effects grade 3 (NCI-CTCAE version 4.0) and breakthrough pain more than 4 episodes/day are point as criteria's to pain multidisciplinary team discussion and consider polymorphism screening (43). Resources are currently limited and a global 
balance is needed for fairer and more equitable treatment worldwide. An investment in the development of laboratory tests for the rapid identification of these polymorphisms is still an unmet need.

\section{Acknowledgements}

The authors would like to thank Miss Luísa Ribeiro from the library services of Francisco Gentil Portuguese Institute of Oncology (Porto, Portugal).

\section{Funding}

The present study was supported as a part of a project selected as an Internal Clinical Research Project of Francisco Gentil Portuguese Institute of Oncology, Porto, Portugal (grant nos. Bulletin 8/2017 and CI-IPOP 70/2017).

\section{Availability of data and materials}

The datasets used and/or analyzed during the current study are available from the corresponding author on reasonable request.

\section{Authors' contributions}

$\mathrm{CV}$ obtained and assessed the data for this review, proposed the levels of evidence and recommended strengths. RF, DP and RM interpreted the results of the study, the levels of evidence and the recommended strengths, and wrote the article. The final manuscript was reviewed and approved by all authors.

\section{Ethics approval and consent to participate}

Not applicable.

\section{Patient consent for publication}

Not applicable.

\section{Competing interests}

The authors declare that they have no competing interests.

\section{References}

1. De Gregori S, Minella CE, De Gregori M, Tinelli C, Ranzani GN, Govoni S, Allegri M and Regazzi M: Clinical pharmacokinetics of morphine and its metabolites during morphine dose titration for chronic cancer pain. Ther Drug Monit 36: 335-344, 2014.

2. O'Brien T, Christrup LL, Drewes AM, Fallon MT, Kress HG, McQuay HJ, Mikus G, Morlion BJ, Perez-Cajaraville J, Pogatzki-Zahn E, et al: European pain federation position paper on appropriate opioid use in chronic pain management. Eur J Pain 21: 3-19, 2017.

3. Leppert W: CYP2D6 in the metabolism of opioids for mild to moderate pain. Pharmacology 87: 274-285, 2011.

4. Kuip EJ, Zandvliet ML, Koolen SL, Mathijssen RH and van der Rijt CC: A review of factors explaining variability in fentanyl pharmacokinetics; focus on implications for cancer patients. Br J Clin Pharmacol 83: 294-313, 2017.

5. Li Y, Jackson KA, Slon B, Hardy JR, Franco M, William L, Poon P, Coller JK, Hutchinson MR, Currow DC and Somogyi AA CYP2B6*6 allele and age substantially reduce steady-state ketamine clearance in chronic pain patients: Impact on adverse effects. Br J Clin Pharmacol 80: 276-284, 2015.
6. Smith HS: Opioid metabolism. Mayo Clin Proc 84: 613-624, 2009.

7. Droney J, Riley J and Ross JR: Evolving knowledge of opioid genetics in cancer pain. Clin Oncol (R Coll Radiol) 23: 418-428, 2011.

8. Ross JR, Rutter D, Welsh K, Joel SP, Goller K, Wells AU, Du Bois R and Riley J: Clinical response to morphine in cancer patients and genetic variation in candidate genes. Pharmacogenomics J 5: 324-336, 2005.

9. Diatchenko L, Robinson JE and Maixner W: Elucidation of mu-Opioid Gene Structure: How genetics can help predict responses to opioids. Eur J Pain Suppl 5: 433-438, 2011.

10. Klepstad P,Rakvåg TT,KaasaS,Holthe M,Dale O,Borchgrevink PC, Baar C, Vikan T, Krokan HE and Skorpen F: The 118 A > G polymorphism in the human mu-opioid receptor gene may increase morphine requirements in patients with pain caused by malignant disease. Acta Anaesthesiol Scand 48: 1232-1239, 2004.

11. De Gregori M, Garbin G, De Gregori S, Minella CE, Bugada D, Lisa A, Govoni S, Regazzi M, Allegri M and Ranzani GN: Genetic variability at COMT but not at OPRM1 and UGT2B7 loci modulates morphine analgesic response in acute postoperative pain. Eur J Clin Pharmacol 69: 1651-1658, 2013.

12. Reyes-Gibby CC, Shete S, Rakvåg T, Bhat SV, Skorpen F, Bruera E, Kaasa S and Klepstad P: Exploring joint effects of genes and the clinical efficacy of morphine for cancer pain: OPRM1 and COMT gene. Pain 130: 25-30, 2007.

13. Rakvag TT, Ross JR, Sato H, Skorpen F, Kaasa S and Klepstad P: Genetic variation in the catechol-O-methyltransferase (COMT) gene and morphine requirements in cancer patients with pain. Mol Pain 4: 64, 2008.

14. Ross JR, Riley J, Taegetmeyer AB, Sato H, Gretton S, du Bois RM and Welsh KI: Genetic variation and response to morphine in cancer patients: Catechol-O-methyltransferase and multidrug resistance-1 gene polymorphisms are associated with central side effects. Cancer 112: 1390-1403, 2008.

15. SORT: The strength-of-recommendation taxonomy. Am Fam Physician 71: 19-20, 2005.

16. de Stoutz ND, Bruera E and Suarez-Almazo M: Opioid rotation for toxicity reduction in terminal cancer patients. J Pain Symptom Manage 10: 378-384, 1995.

17. Benedetti C, Brock C, Cleeland C, Coyle N, Dubé JE, Ferrell B, Hassenbusch S III, Janjan NA, Lema MJ, Levy MH, et al: NCCN practice guidelines for cancer pain. Oncology (Williston Park) 14: 135-150, 2000.

18. Scottish Intercollegiate Guidelines Network (SIGN). Management of chronic pain. Edinburgh: SIGN; 2013. (SIGN publication no. 136). [December, 2013]. Available from URL: http://www.sign.ac.uk

19. Ripamonti CI, Santini D, Maranzano E, Berti M and Roila F; ESMO Guidelines Working Group: Management of cancer pain: ESMO clinical practice guidelines. Ann Oncol 23 (Suppl 7): vii139-vii154, 2012.

20. Pigni A, Brunelli C, Gibbins J, Hanks G, Deconno F, Kaasa S, Klepstad P, Radbruch L and Caraceni A: Content development for EUROPEAN GUIDELINES on the use of opioids for cancer pain: A systematic review and expert consensus study. Minerva Anestesiol 76: 833-843, 2010.

21. King S, Forbes K, Hanks GW, Ferro CJ and Chambers EJ: A systematic review of the use of opioid medication for those with moderate to severe cancer pain and renal impairment: A European Palliative Care Research Collaborative opioid guidelines project. Palliat Med 25: 525-552, 2011.

22. Burton AW and Cleeland CS: Cancer pain: Progress since the WHO guidelines. Pain Pract 1: 236-242, 2001.

23. Ventafridda V, Saita L, Ripamonti C and De Conno F: WHO guidelines for the use of analgesics in cancer pain. Int $\mathrm{J}$ Tissue React 7: 93-96, 1985.

24. Schug SA, Zech D and Dörr U: Cancer pain management according to WHO analgesic guidelines. J Pain Symptom Manage 5: 27-32, 1990.

25. Ripamonti C and Giusti R: 21st expert committee on the selection and use of essential medicines: Opioid analgesics for cancer pain: fentanyl, methadone, tramadol-EML (EML Opioids for cancer pain: A comparative overview). OMS. Available from URL: Accessed Jan, 2018.

26. Schug SA and Chandrasena C: Pain management of the cancer patient. Expert Opin Pharmacother 16: 5-15, 2015.

27. Dowell D, Haegerich TM and Chou R: CDC guideline for prescribing opioids for chronic pain-united states, 2016. JAMA 315: 1624-1645, 2016. 
28. Busse JW, Craigie S, Juurlink DN, Buckley DN, Wang L, Couban RJ, Agoritsas T, Akl EA, Carrasco-Labra A, Cooper L, et al: Guideline for opioid therapy and chronic noncancer pain. CMAJ 189: E659-E666, 2017.

29. Lucey M; Guideline Development Group: Pharmacological Management of Cancer Pain in Adults-National Clinical Guideline No. 9. Ireland, 2015.

30. Jara C, Del Barco S, Grávalos C, Hoyos S, Hernández B, Muñoz M, Quintanar T, Meana JA, Rodriguez C and de Las Peñas R: SEOM clinical guideline for treatment of cancer pain (2017). Clin Transl Oncol 20: 97-107, 2018.

31. Nielsen LM, Olesen AE, Branford R, Christrup LL, Sato H and Drewes AM: Association between human pain-related genotypes and variability in opioid analgesia: An updated review. Pain Pract 15: 580-594, 2015.

32. Shi Q, Cleeland CS, Klepstad P, Miaskowski C and Pedersen NL; GeneQOL Consortium: Biological pathways and genetic variables involved in pain. Qual Life Res 19: 1407-1417, 2010.

33. Hwang IC, Park JY, Myung SK, Ahn HY, Fukuda K and Liao Q: OPRM1 A118G gene variant and postoperative opioid requirement: A systematic review and meta-analysis. Anesthesiology 121: 825-834, 2014.

34. Chou WY, Wang CH, Liu PH, Liu CC, Tseng CC and Jawan B: Human opioid receptor $\mathrm{A} 118 \mathrm{G}$ polymorphism affects intravenous patient-controlled analgesia morphine consumption after total abdominal hysterectomy. Anesthesiology 105: 334-337, 2006.

35. Nagashima M, Katoh R, Sato Y, Tagami M, Kasai S and Ikeda K: Is there genetic polymorphism evidence for individual human sensitivity to opiates? Curr Pain Headache Rep 11: 115-123, 2007.

36. Tammimäki A and Männistö PT: Catechol-O-methyltransferase gene polymorphism and chronic human pain: A systematic review and meta-analysis. Pharmacogenet Genomics 22: 673-691, 2012.

37. Kluetz PG, Chingos DT, Basch EM and Mitchell SA: Patient-reported outcomes in cancer clinical trials: Measuring symptomatic adverse events with the national cancer institute's patient-reported outcomes version of the common terminology criteria for adverse events (PRO-CTCAE). Am Soc Clin Onco Educ Book 35: 67-73, 2016.

38. Andersen RL, Johnson DJ and Patel JN: Personalizing supportive care in oncology patients using pharmacogenetic-driven treatment pathways. Pharmacogenomics 17: 417-434, 2016.
39. Slatkin NE: Opioid switching and rotation in primary care: Implementation and clinical utility. Curr Med Res Opin 25: 2133-2150, 2009.

40. Zhu G, Wang L, Guo H, Lu L, Yang S, Wang T, Guo H, Wang H, Min J, Yang K, et al: DNA repair genes XRCC1 and ERCC1 polymorphisms and the risk of sporadic breast cancer in Han women in the Gansu Province of China. Genet Test Mol Biomarkers 19: 387-393, 2015.

41. Lu L, Katsaros D, Mayne ST, Risch HA, Benedetto C, Canuto EM and Yu H: Functional study of risk loci of stem cell-associated gene lin-28B and associations with disease survival outcomes in epithelial ovarian cancer. Carcinogenesis 33: 2119-2125, 2012.

42. Lu L, Risch E, Deng Q, Biglia N, Picardo E, Katsaros D and $\mathrm{Yu} \mathrm{H}$ : An insulin-like growth factor-II intronic variant affects local DNA conformation and ovarian cancer survival. Carcinogenesis 34: 2024-2030, 2013.

43. Sloan PA, Donnelly MB, Schwartz RW and Sloan DA: Cancer pain assessment and management by housestaff. Pain 67: 475-481, 1996.

44. De Gregori M, Diatchenko L, Belfer I and Allegri M: OPRM1 receptor as new biomarker to help the prediction of post mastectomy pain and recurrence in breast cancer. Minerva Anestesiol 81: 894-900, 2015.

45. Andersen S and Skorpen F: Variation in the COMT gene: Implications for pain perception and pain treatment. Pharmacogenomics 10: 669-684, 2009.

46. Fladvad T, Fayers P, Skorpen F, Kaasa S and Klepstad P: Lack of association between genetic variability and multiple pain-related outcomes in a large cohort of patients with advanced cancer: The European Pharmacogenetic Opioid Study (EPOS). BMJ Support Palliat Care 2: 351-355, 2012

47. Wang G, Zhang H, He F and Fang X: Effect of the CYP2D6*10 C188T polymorphism on postoperative tramadol analgesia in a Chinese population. Eur J Clin Pharmacol 62: 927-931, 2006.

48. Holthe M, Klepstad P, Zahlsen K, Borchgrevink PC, Hagen L, Dale O, Kaasa S, Krokan HE and Skorpen F: Morphine glucuronide-to-morphine plasma ratios are unaffected by the UGT2B7 $\mathrm{H} 268 \mathrm{Y}$ and UGT1A1*28 polymorphisms in cancer patients on chronic morphine therapy. Eur J Clin Pharmacol 58: 353-356, 2002.

This work is licensed under a Creative Commons

Attribution-NonCommercial-NoDerivatives 4.0 International (CC BY-NC-ND 4.0) License. 